\title{
Tuberculosis elimination: where are we now?
}

\author{
Alberto Matteelli ${ }^{1}$, Adrian Rendon ${ }^{2}$, Simon Tiberi $^{3}$, Seif Al-Abri ${ }^{4}$, \\ Constantia Voniatis ${ }^{5}$, Anna Cristina C. Carvalho ${ }^{6}$, Rosella Centis (1) 7 , \\ Lia D’Ambrosio (107, ${ }^{7}$, Dina Visca?, Antonio Spanevello ${ }^{9,10}$ and \\ Giovanni Battista Migliori (i) ${ }^{7}$
}

\begin{abstract}
Affiliations: ${ }^{1}$ University Dept of Infectious and Tropical Diseases, WHO Collaborating Centre for TB/HIV coinfection and for TB elimination, University of Brescia and Brescia Spedali Civili General Hospital, Brescia, Italy. ${ }^{2}$ Centro de Investigación, Prevención y Tratamiento de Infecciones Respiratorias, Hospital Universitario de Monterrey, Monterrey, México. ${ }^{3}$ Division of Infection, Royal London Hospital, Barts Health NHS Trust, London, UK. ${ }^{4}$ Ministry of Health, Muscat, Oman. ${ }^{5}$ Clinical Laboratories, Ministry of Health, Nicosia, Cyprus. ${ }^{6}$ Laboratory of Innovations in Therapies, Education and Bioproducts (LITEB), Oswaldo Cruz Institute (IOC), FioCruz, Rio de Janeiro, Brazil. "World Health Organization Collaborating Centre for Tuberculosis and Lung Diseases, Maugeri Care and Research Institute, IRCCS, Tradate, Italy. ${ }^{8}$ Public Health Consulting Group, Lugano, Switzerland. ${ }^{9}$ Pneumology Dept, Maugeri Care and Research Institute, Tradate, Italy. ${ }^{10}$ Dept of Clinical and Experimental Medicine, University of Insubria, Varese, Italy
\end{abstract}

Correspondence: Giovanni Battista Migliori, World Health Organization Collaborating Centre for Tuberculosis and Lung Diseases, Maugeri Care and Research Institute, IRCCS, Via Roncaccio 16, 21049, Tradate, Italy. E-mail: giovannibattista.migliori囚icsmaugeri.it

@ERSpublications

The theoretical principles and the available examples of countries moving towards TB elimination are described in this article http://ow.ly/q8wN30k9UOW

Cite this article as: Matteelli A, Rendon A, Tiberi S, et al. Tuberculosis elimination: where are we now? Eur Respir Rev 2018; 27: 180035 [https://doi.org/10.1183/16000617.0035-2018].

ABSTRACT Tuberculosis (TB) still represents a major public health issue in spite of the significant impact of the efforts made by the World Health Organization (WHO) and partners to improve its control. In 2014 WHO launched a new global strategy (End TB) with a vision of a world free of TB, and a 2035 goal of TB elimination (defined as less than one incident case per million). The aim of this article is to summarise the theoretical bases of the End TB Strategy and to analyse progresses and persistent obstacles on the way to TB elimination.

The evolution of the WHO recommended strategies of TB control (Directly Observed Therapy, Short Course (DOTS), Stop TB and End TB) are described and the concept of TB elimination is discussed. Furthermore, the eight core activities recently proposed by WHO as the milestones to achieve $\mathrm{TB}$ elimination are discussed in detail. Finally, the recently published experiences of Cyprus and Oman on their way towards TB elimination are described, together with the regional experience of Latin America.

New prevention, diagnostic and treatment tools are also necessary to increase the speed of the present TB incidence decline.

\section{Introduction}

Tuberculosis (TB) still represents a major public health issue in spite of the significant impact of the efforts made by the World Health Organization (WHO) and partners to improve its control [1].

The WHO estimates that 10.4 million individuals became ill with TB and 1.7 million died in 2016. Despite the fall in mortality rate of $3 \%$ per year, TB remains the ninth leading cause of death worldwide, counting 1.3 million TB deaths among HIV-negative people and almost 400000 deaths among HIV-positive people [1].

Provenance: Commissioned article, peer reviewed.

Received: March 302018 | Accepted after revision: May 152018

Copyright CERS 2018. ERR articles are open access and distributed under the terms of the Creative Commons Attribution Non-Commercial Licence 4.0 . 
Severe critical challenges can be identified that hamper effective TB control. Persistently high TB mortality can be reduced by earlier diagnosis and treatment. For as many as 3 million estimated TB cases we have no notification, raising serious concern on optimal clinical and public health management. This is mainly attributed to incomplete compliance of private providers in some countries, while economic barriers to primary care access might determine underdiagnosed TB in low-income countries [1]. Multidrug-resistant (MDR)-TB is another significant challenge. In 2016 there were 600000 new cases with resistance to rifampicin, of which 490000 had MDR-TB (e.g. resistance to at least isoniazid and rifampicin), including $6.2 \%$ of extensively drug-resistant (XDR)-TB (e.g. MDR-TB with resistance to fluoroquinolones and one second-line injectable). Drug resistance implies low treatment success rates (e.g. 54\% for MDR-/ rifampicin-resistant TB and 30\% for XDR-TB) [1-3]. Countries with the highest MDR-TB burden (47\% of the global total) are India, China and the Russian Federation [1].

In this bleak scenario, WHO launched a new global strategy (End TB) with a vision of a world free from TB, and a 2035 goal of TB elimination (i.e. less than one incident case per million) [4]. In this article we summarise the theoretical bases of the End TB Strategy and we analyse progresses and persistent obstacles on the way to TB elimination.

\section{A summary of the WHO strategies \\ The DOTS Strategy}

The WHO launched the DOTS (Directly Observed Therapy, Short Course) Strategy in 1995, the first comprehensive public health approach to TB aimed at having a significant impact on the TB epidemic $[4,5]$. Consisting of five core elements (figure 1), it strengthened the capacity of national programmes to diagnose and treat TB cases, with a clear focus on those who were able to transmit the infection [6-9]: sputum smear positive cases were prioritised for diagnosis and treatment with standardised short-course chemotherapy regimens. The acronym chosen was a thoughtful decision to emphasise the need to adopt the 6-month anti-TB regimen (known as category 1 regimen) to treat new $\mathrm{TB}$ cases. The regimen consisted of a 2-month initial phase of isoniazid, rifampicin, pyrazinamide and ethambutol followed by a 4-month continuation phase including isoniazid and rifampicin [10]. The importance of ensuring continuous drug supply was fully recognised.

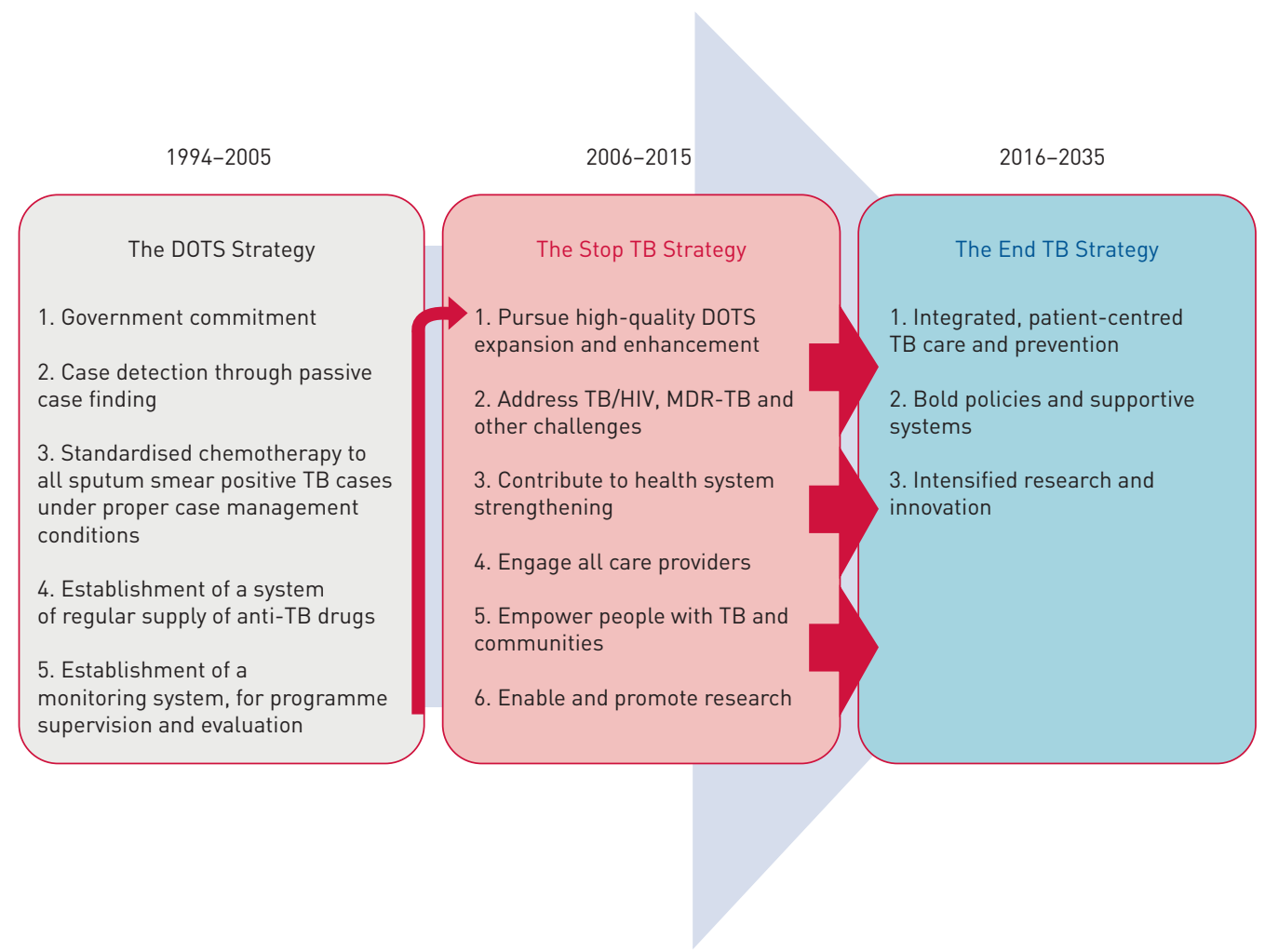

FIGURE 1 Evolution of global anti-tuberculosis (TB) strategies. DOTS: directly observed therapy, short course; MDR: multidrug resistant. 
The strategy introduced case recording and reporting as an essential element to evaluate the entire intervention (figure 1). Government commitment was a clear pre-condition to make the strategy effective.

\section{The Stop TB Strategy}

In 2006 due to several emerging new priority interventions, the WHO launched the Stop TB Strategy (figure 1), which was organised into six elements $[4,5]$.

The first element endorsed the continuation of the DOTS package as a whole, within an evolving framework. Sustained financing was used in an era of greater attention to sustainability; quality-assured bacteriology to go beyond sputum smear microscopy (the focus was on culture and universal drug susceptibility testing); patient support (a move towards a patient-centred vision with many implications [11-15]); monitoring and evaluation; and impact measurement. The new elements of the strategy were represented by targeted measures against MDR-TB and TB/HIV co-infection, with new attention given to the private sector (up to half of the TB cases are served by the private sector in the Indian sub-continent and in other settings), as well as patients and communities. The concepts of "health system strengthening" and "enhancing research" were also new.

\section{The End TB Strategy}

The vision radically evolved in the End TB Strategy (figure 1) [4, 5, 16-20] launched by WHO in 2014 and consisting of three pillars (tables 1 and 2). The first pillar summarised the core concepts of the previous strategies related to diagnosis and treatment of $\mathrm{TB}$, while introducing for the first time the concept of prevention, mainly based on the identification and treatment of individuals with latent $\mathrm{TB}$ infection (LTBI) [20-22].

The second pillar summarised all inter-ministerial activities that are at a level above the national TB control programme teams and are relevant to impact TB control and elimination, starting with social determinants (i.e. poverty alleviation) and ensuring universal health coverage, vital registration, rational use of drugs, infection control and social protection [4, 8, 20, 23-26].

\section{A paradigm shift: from TB control to TB elimination}

$\mathrm{TB}$ control is classically seen as the strategy aimed at diagnosing and rapidly rendering infectious cases non-infectious, in order to break the chain of transmission [9, 18, 27, 28].

The TB elimination strategy broadens the concept of TB control to identify and treat the pool of latently infected individuals from which future TB cases will be generated $[9,18,27,28]$.

TB elimination, defined as less than one TB case per million population, is seen as a scenario where TB is not eradicated but rather kept at such a low level that it no longer constitutes a public health problem [18].

TB eradication (e.g. the complete disappearance of the disease) is not considered possible considering the dynamic model of its transmission (large "reservoir" of infected individuals, of whom only a low proportion develops the disease) and the absence of a vaccine able to protect all individuals vaccinated.

\section{TABLE 1 The End TB strategy pillars}

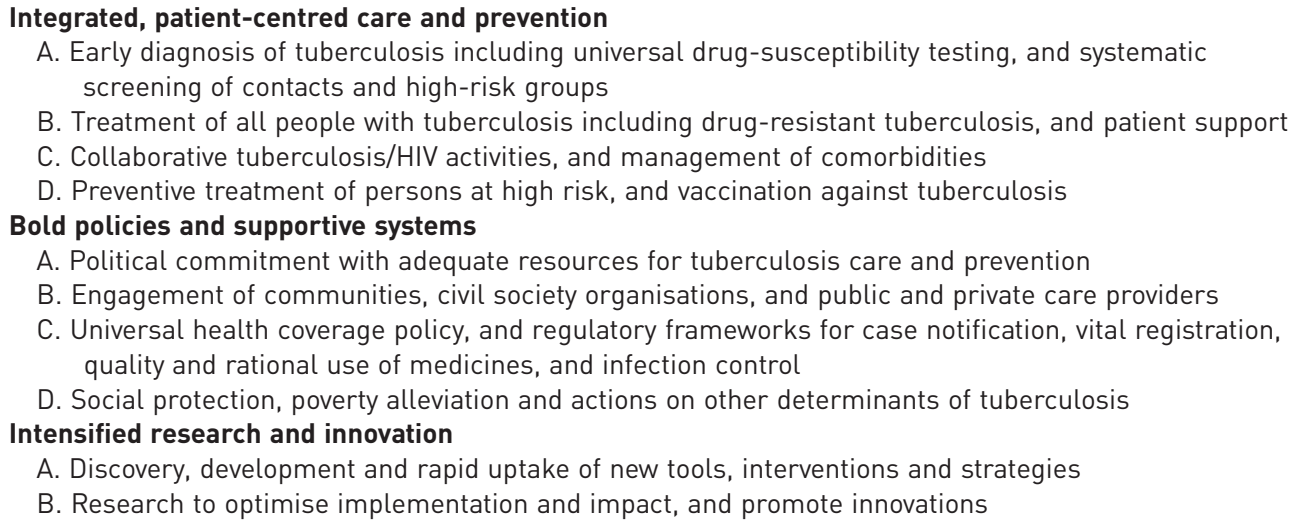

A. Political commitment with adequate resources for tuberculosis care and prevention

B. Engagement of communities, civil society organisations, and public and private care providers

C. Universal health coverage policy, and regulatory frameworks for case notification, vital registration, quality and rational use of medicines, and infection control

D. Social protection, poverty alleviation and actions on other determinants of tuberculosis

Intensified research and innovation

A. Discovery, development and rapid uptake of new tools, interventions and strategies

B. Research to optimise implementation and impact, and promote innovations

Data from [4] 
TABLE 2 Tuberculosis (TB) elimination priority action areas

Priority action areas to reach TB elimination

Global strategy

pillars and

components

1. A-D
2. A-D
1. A-D
2. B-D
1. A-D
2. B-D
1. A, D
1. A-D
2. A-D
3. A-B
2. A-C
3. A-B
1-3

1. Ensure political commitment, funding and stewardship for planning and essential services of high quality

2. Address the most vulnerable and hard-to-reach groups

3. Address special needs of migrants and cross-border issues

4. Undertake screening for active TB and LTBI in TB contacts and selected high-risk groups, and provide appropriate treatment

5. Optimise the prevention and care of drug-resistant TB

6. Ensure continued surveillance, programme monitoring and evaluation and case-based data management

7. Invest in research and new tools

8. Support global TB prevention, care and control

LTBI: latent TB infection. Data from [4].

Strong support for the elimination hypothesis is derived from mathematical modelling. A famous modelling study by Dye et al. [29] showed the potential major impact of preventive interventions by 2050 . In particular the study findings suggest that very low incidence rates approaching one TB case per million can be reached only by combining rapid diagnosis and effective treatment of both active TB and LTBI cases [29]. Importantly, the impact of social protection and poverty reduction on TB incidence has been recently underlined $[30,31]$.

Models predict that elimination of TB can be reached within the next few decades and can discern what the relevant contribution of a new vaccine, new (shorter and better tolerated) regimens to manage TB and LTBI and a new point of care diagnostic test would be $[32,33]$. Diagnosis and treatment of LTBI outperforms other interventions in these scenarios.

Other models describe the possible (and epidemiologically plausible) evolution of the disease from 2015 to 2050 (figure 2) [34]. At present the annual decline in TB incidence is rather slow (in the order of $1.5 \%$ globally), as it results from a composition of the $7-10 \%$ decline observed in low TB incidence countries, the $5 \%$ observed in intermediate incidence countries (e.g. China) and a much more modest downward trend in high TB incidence countries [1]. The figure shows potential reduction based on different scenarios: best use of available tools (Xpert and new generation Inno-Lipa rapid diagnostics [35-39], shorter regimen and new/repurposed drugs [40-70]) followed by rapid progression following the introduction of new tools and drugs from 2025 onwards.

FIGURE 2 Projected acceleration in the decline of global tuberculosis (TB) incidence rates to target levels. Reproduced from [34] with permission.

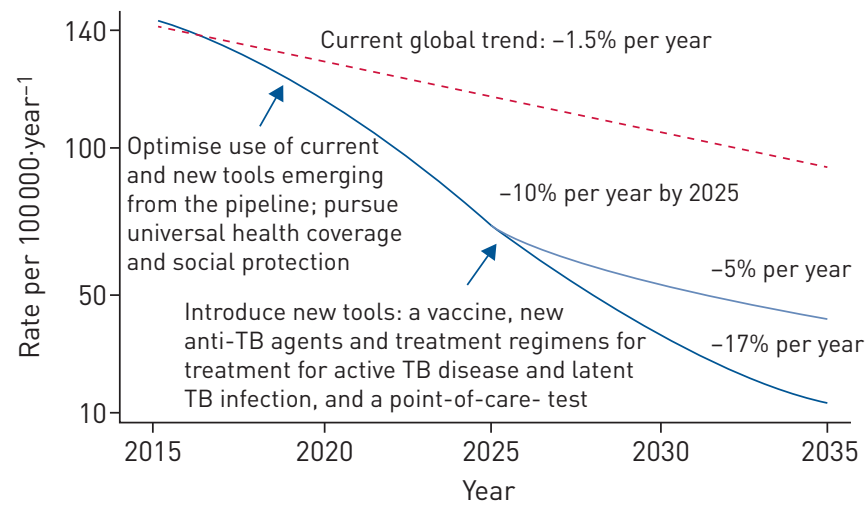


Low TB incidence countries (those with $<10$ notified cases per 100000 population $[18,19]$ ) fall into an epidemiological situation where TB elimination is particularly conceivable and could be reached by 2050 , passing through a pre-elimination phase (defined as $<10$ cases per million population) by 2035 [18, 71, 72] . Low TB incidence countries have been invited to take action and implement adequate interventions to make this possible.

The eight core activities to achieve TB elimination in low TB incidence countries

The WHO and the European Respiratory Society developed a framework and identified eight core activities to achieve TB elimination in countries at low incidence of TB (originally defined as those with $<10$ cases per 100000 population) $[18,73]$.

\section{Ensure political commitment, funding and stewardship}

Although involvement of the private sector is crucial (it needs to be linked to the national TB programme, so as to ensure that cases are notified and managed according to the standard, e.g. using validated diagnostics and WHO recommended treatment regimens), TB control and elimination cannot be reached in the absence of governmental commitment, funding and stewardship [4]. National TB programmes (which work within the Ministries of Health [31]) should ensure that diagnosis, treatment and prevention of TB are correctly implemented through an adequate legal framework. It is necessary to ensure that: TB cases are duly notified and outcomes of treatment analysed; validated diagnostic algorithms are used (e.g. by regulating reimbursements to both private and public health providers); and only quality drugs are available for use or that antibiotics are prescribed according to a given hierarchy (thus ensuring rational use of drugs and preventing selection of drug resistance). Beyond the legal framework, funding and stewardship are core pre-conditions to pursue TB elimination [4, 18, 74]. Even in low TB incidence, high-income countries funding can be inadequate, particularly when vertical TB control programmes have been dismantled (because they are not cost-effective) and TB services decentralised. Furthermore, the regionalisation of health services might complicate both coordination and funding of core activities [75]. In summary, it is of paramount importance to keep a central coordination level of anti-TB activities even within a decentralised system, where it is clear "who is doing what" both on the prevention and clinical side, and keep an adequate funding stream for the eight activities described above [18]. Obviously, with a decreasing number of cases it becomes increasingly difficult to keep $\mathrm{TB}$ a high priority on the governmental agenda [75].

The "WHO Global Ministerial Conference on Ending TB in the Sustainable Development Era: a Multisectoral Response" was conducted in 2017 in Moscow, Russia, to accelerate implementation of the WHO End TB Strategy [74]. Its product, the "Moscow declaration", represents a fundamental milestone [74]. It calls for action at global and regional levels for four key areas. 1) Universal health coverage by strengthening health systems and improving access to people-centred TB prevention and care, ensuring no one is left behind; 2) mobilisation of sufficient and sustainable financing through increased domestic and international investments to close gaps in implementation and research; 3) advancing research and development of new tools to diagnose, treat and prevent TB; and 4) building accountability through a framework to track and review progress on ending TB, including multisector approaches [74]. Later in 2018 a UN General Assembly on TB will further contribute to raise the position of TB on the agenda of member states.

\section{Address the vulnerable and hard-to-reach groups}

There is undisputable evidence that $\mathrm{TB}$ is a poverty-related disease that concentrates in the most vulnerable, marginalised and hard-to-reach populations.

Universal and free of costs access to TB services, in the absence of stigma (affecting care-seeking behaviour), has been recommended by WHO and by the main TB guidelines for two main reasons [13, 20, 76-78]. The first reason is a principle, and relates to individual human rights: each human being has equal rights to a healthy life. The second reason is related to the specific characteristic of the disease: the best prevention of TB consists of rapid diagnosis and effective treatment of infectious cases, so that it is in the communities interest to ensure that all individuals with signs and symptoms compatible with TB are diagnosed and treated in a timely manner, even in the absence of health insurance and independently from any other legal or residency requirements [13, 20, 76-79].

Address special need of migrants and cross-border issues

North-South and East-West migrations have been taking place for years [80, 81]. Massive migration from Europe took place in the 19th Century contributing to the development of the USA and several Latin American countries; similar complex flows have been observed elsewhere before and after [82]. 
In 2016, approximately a billion people ( $>14 \%$ of the world's population) were migrants: 250 million international migrants and 763 million internal migrants. Globally, about 65 million of them underwent forced displacement from their homes and 20 million people were considered refugees [83].

From a European perspective, almost 1.8 million migrants crossed the Mediterranean sea between 2014 and 2018 (table 3), with 16000 dying during the trip [84], with many more reaching it by different sea routes. Large waves of refugees over a short period of time (for example over 400000 Syrians sought asylum in Germany in 2015) created obvious management problems [85].

The main reason behind this massive move of populations is poverty and the hope of a better life. Migrants and refugees escape war, famine, natural disasters and persecution, usually making long and dangerous trips. Poverty, malnutrition, crowding and stress favour development and transmission of several diseases, including TB [76-79]. The risk of acquiring TB disease given infection increases in the presence of the risk factors described above $[6,7]$.

TB case notifications in many low-incidence countries are disproportionately higher in migrants, calling for enhanced TB control strategies in these populations to achieve TB elimination. Countries have generally focused on two targeted control strategies, balancing identification of active TB before or soon after arrival in the host country to detect prevalent TB cases, and diagnosis and treatment of LTBI to prevent TB reactivation.

More than a century ago passengers arriving by sea to the USA were asked to climb long and steep stairs carrying their luggage under the observation of custom officers. Those who were smiling or laughing were screened for mental disorders (of which syphilis was a common cause at the time) and those coughing were screened for TB [82].

As of today evidence on optimal and cost-effective ways of screening for TB and LTBI (either before migration, at the port of entry or after reaching the host country) continue to be elusive. The WHO recommends (only conditionally) both TB and LTBI screening among migrants living in low TB burden countries due to reservations about implementation issues, as well as the low quality of evidence of the effectiveness and cost-effectiveness of available tools.

\section{Manage LTBI}

LTBI affects one-third to one-quarter of the population and is the reservoir for the large majority of emerging active TB cases in low incidence countries. The management of LTBI first entered WHO recommendations with the End TB Strategy, as a mainstay of its first pillar and a key element to pursue elimination. Testing and treatment for LTBI should be organised with a programmatic perspective. This implies strong political commitment, adequate funding, and an effective monitoring and evaluation

TABLE 3 Migration flows in the Mediterranean area; sea departures and arrivals 2014-2018

\begin{tabular}{|c|c|c|}
\hline Year" & Arrivals $\mathrm{n}^{\#}$ & Deaths n $(\%)^{\#}$ \\
\hline 2014 & 216054 & $3538(1.6)$ \\
\hline 2015 & 1015078 & $3771(0.4)$ \\
\hline 2016 & 362753 & $5096(1.4)$ \\
\hline 2017 & 172301 & $3139(1.8)$ \\
\hline 2018 & 24042 & $609(2.5)$ \\
\hline Total & 1790228 & $16153(0.9)$ \\
\hline $\begin{array}{l}\text { Top countries of departure from } \\
\text { January } 2017\end{array}$ & $\begin{array}{l}\text { Population departing } \\
\text { from January } 2017\end{array}$ & $\begin{array}{l}\text { Percentage of departures } \\
\text { from January } 2017\end{array}$ \\
\hline Syrian Arab Rep. ${ }^{\Uparrow}$ & 22253 & 11.6 \\
\hline Nigeria $^{+}$ & 18590 & 9.7 \\
\hline Guinea $^{+}$ & 14745 & 7.7 \\
\hline Top countries of arrivals in 2018 & $\begin{array}{l}\text { Population arriving in } \\
\qquad 2018\end{array}$ & $\begin{array}{l}\text { Percentage of arrivals in } \\
2018\end{array}$ \\
\hline Italy\# & 9621 & 40.0 \\
\hline Spain $\S$ & 7114 & 29.6 \\
\hline Greece & 9375 & 39.0 \\
\hline
\end{tabular}

\#: data from May 8, 2018; ๆ: data from April 30, 2018; ${ }^{+}$: data from March 31, 2018; ${ }^{\S}$ : data from May 7 , 2018; ${ }^{f}$ : data from May 6, 2018. Data from [84]. 
system. In the recently updated LTBI guidelines [22], the WHO unified pre-existing multiple documents and fragmented recommendations thus providing universal guidance. Currently, available LTBI diagnostic tests are poorly predictive, and treatment regimens are long and poorly tolerated. In this situation, the harm and benefit trade-off is unfavourable in most populations, except those with a very high risk of reactivation. According to $\mathrm{WHO}$, people living with $\mathrm{HIV}$, children aged $<5$ years who are household contacts of a contagious TB case, and people with clinical conditions such as silicosis, diseases requiring anti-tumour necrosis factor treatment, dialysis, and transplantation, should be primarily targeted in all epidemiological settings. In high resource and low TB incidence settings, additional at-risk populations should also be the targeted for systematic LTBI testing and treatment, including HIV-negative children aged $\geqslant 5$ years, adolescents and adults who are household contacts of people with bacteriologically confirmed pulmonary TB cases, immigrants from highly endemic areas, prisoners and drug users [22]. In specific settings (e.g. Mexico), as $30-40 \%$ of the TB cases have diabetes, collaboration with diabetes clinics represents an opportunity to manage LTBI in this risk group [86, 87].

Among the important challenges for the countries approaching the TB pre-elimination phase we underline the implementation of new generation diagnostics (test with higher prediction capacity on future disease) and safer and shorter treatment regimens (the rifapentine/isoniazid weekly regimen for 12 weeks or the ultra-short one month daily rifapentine/isoniazid regimen) $[22,88,89]$.

Optimise prevention and care of MDR-TB

MDR-TB is omnipresent and represents an emergency in some settings [1]. In Europe, hot spots are located in the former Soviet Union countries, where $>30 \%$ of new TB cases are MDR.

Treating MDR- and XDR-TB is long (almost 2 years versus 6 months for a drug-susceptible case), difficult (second-line anti-TB drugs are very toxic) and very expensive (the cost of treating XDR-TB might exceed $€ 100000$ in Europe, while a regimen for drug susceptible cases costs less than €20) [64, 90, 91].

A plethora of causes favours selection of drug resistant mutants, the common denominator being incorrect treatment. Any cause of incorrect treatment (from poor-quality drugs to poorly designed regimens i.e. drug selection, dosage and duration) would favour increasing prevalence of drug resistance [6, 7]. Therefore, the best prevention of drug resistance is rapid diagnosis and effective treatment of drug susceptible cases, although treatment of those already affected by MDR-/XDR-TB is also important as they can further propagate drug resistance.

Under this perspective, adequate infection control practices in healthcare facilities are of paramount importance in limiting transmission to healthcare staff, patients and visitors, taking into account that the "dangerous" TB cases are those that are undetected, including the MDR-/XDR-TB cases not recognised as such and treated as drug-susceptible cases [26, 92].

Recent evidence suggests that correct treatment is extremely effective in rendering the patient rapidly non-infectious. Today, the availability of rapid diagnostic methods allows us to identify, within a few hours, whether the strain is susceptible or resistant to drugs and, therefore, enables appropriate treatment accordingly [32, 33, 35-37]. Unfortunately, the practice of treating patients with the standard regimen for new cases ("blindly" as far as drug resistance is concerned) and considering drug susceptibility testing only when treatment failure occurs is still common in many settings [93].

Last but not least, treatment adherence is of paramount importance in preventing the emergence of resistance. Therefore, any initiative in the direction of supporting adherence with a "patient-centred approach" will contribute to reduce the probability that new drug resistance will occur [13].

In summary, national TB programmes need to ensure that key interventions including systematic use of rapid genotypic methods and prescription of effective regimens (including the shorter ones when applicable) within a patient-centred approach are implemented.

Improve surveillance, monitoring and evaluation and case-based data management

The importance of evaluating treatment outcomes and the performance of TB control programmes was emphasised in the DOTS strategy, and further developed in parallel with the evolution of the WHO-recommended strategies of TB control. Karel Styblo conceptualised the International Union Against Tuberculosis model of TB control programmes in Tanzania during the 1980s, identifying a few core variables to collect so as to monitor the programme, including treatment outcomes, based on a simple paper-based system that was also likely to work in disadvantaged settings [4, 9, 94].

With improved technological development and accessibility, there is consensus that computerised, individual data surveillance systems are necessary in all countries [95], allowing more precise and rapid monitoring and evaluation of the programme performances at the regional and national level; in addition 
to supporting a better clinical management of individual patients at the peripheral level. The need for comprehensive individual information is more stringent for MDR-TB cases, as their management is more complicated. An adequate legal framework is necessary to sustain the improvement of surveillance systems at the national level [4]. A greater emphasis on cross-border management of cases and national programme cooperation will be key in managing migrants with active TB more effectively and efficiently. The End TB Strategy calls for increased efforts to evaluate the programmatic impact on the TB epidemic with the hope to monitor the trajectory towards elimination in low TB incidence countries.

\section{Invest in research}

Research is essential at different levels. New vaccines, diagnostics and drugs/regimens are necessary to pursue TB elimination [18]. However, research has a much wider scope, including operational research on how better to introduce and use new tools, and improving specific aspects of the TB control and elimination programme. Improved data at the national and global level, as discussed above, are key to allow these operational research projects to be successful.

This cannot happen if the countries fail in developing country-specific, priority oriented and funded national TB research plans [4].

\section{Support global TB prevention, care and control}

$\mathrm{TB}$ does not respect borders and international collaboration is necessary to achieve $\mathrm{TB}$ control and hopefully elimination [1, 76-79]. As discussed above, much was done to develop a framework for global data collection (allowing WHO to publish its annual TB global report) and to implement a mechanism funding TB control efforts in economically disadvantaged countries (the Global Fund). However, the funding gap is still consistent and more needs to be done through a better collaboration among all stakeholders, including the private sector [1].

\section{The existing country experiences \\ Cyprus}

A recent study has investigated the progress achieved by Cyprus towards TB elimination [96]. Cyprus has a population of about 1 million, and delivers a quality health service and TB programme. Although a formal TB elimination plan does not exist, the eight core activities described above are implemented.

TB diagnosis and treatment are available free of charge. It was estimated that $85 \%$ of the $301 \mathrm{~TB}$ patients diagnosed in 2011 were managed as outpatients, paying a nominal fee for these services.

A single, well equipped MDR-TB unit is available, all anti-TB drugs are available and the laboratory undergoes regular external quality control.

LTBI screening and contact tracing are regularly performed in risk groups, particularly in contacts of index cases and migrants. The 6-9 month isoniazid regimen is regularly used, although treatment completion rates were not recorded at the time the study was performed.

Impact indicators are summarised in figure 3 [96]. As expected, in similar countries the numbers are small. The overall TB notification rate calculated per million population decreased from 85 in 1980 to a historical minimum of 28 in 2002. Among infectious (sputum smear positive) TB cases, the decline was from 24 to 11 cases per million.

The balance between individuals born in Cyprus and abroad was reached in 2003; in the following years the proportion of TB cases among migrants progressively increased, reaching a plateau above $80 \%$ in 2007 .

TB notifications and rates (all forms) declined progressively among native Cypriots from 31 cases per million in 1996 to six cases per million in 2004, then reaching a plateau around 7-8 cases per million. Interestingly, the decline of infectious cases almost reached the TB elimination threshold (1 case per million), with 2 cases per million in 2005.

Among foreign-born individuals, the rates of notified cases increased to 54 cases overall and 9 cases per million (sputum smear positive) in 2009.

The TB trends in Cyprus demonstrate that adequate TB control measures allowed an annual average decline of $9.4 \%$ between 1997 and 2002 (from 46 to 20 cases) to be reached. A mild increase was then observed, due to the contribution from the immigrant population.

Cyprus reported and estimated a case-detection rate of 100\% in 2012 (and more than 87\% since 1990), with a rapid capacity to diagnose cases (diagnostic delay: 2-4 days) and high success rates (exceeding $85 \%$ since 2006). 
FIGURE 3 Tuberculosis (TB) cases per million population (1980-2012) in Cyprus. $\mathrm{SS}^{+}$: sputum smear positive. Reproduced from [96] with permission.

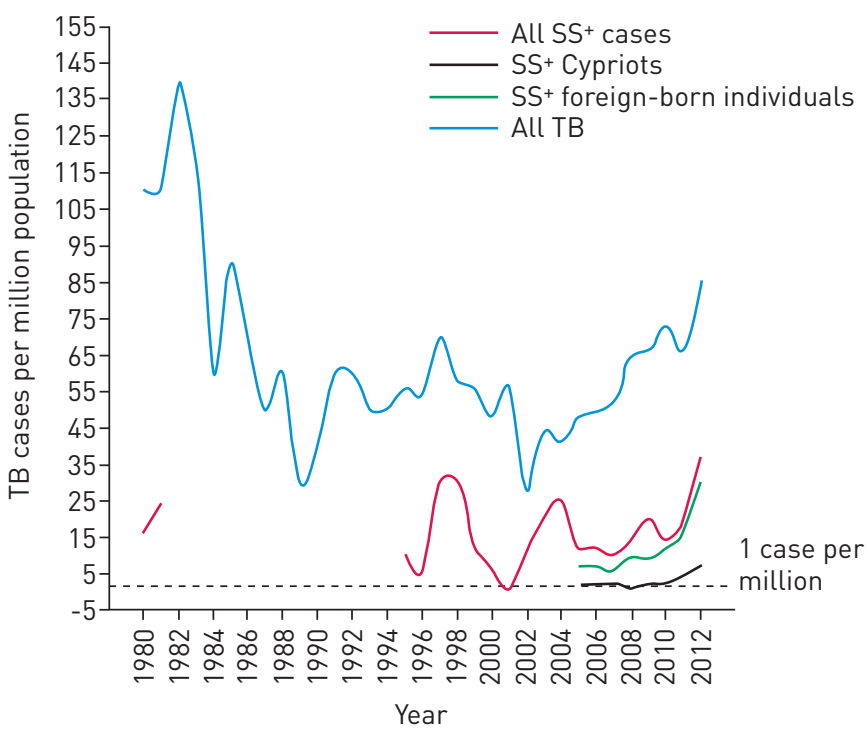

The low MDR-TB prevalence (4.4\% including both new and re-treatment cases together for a total of 10 cases diagnosed between 2005 and 2012) testifies the quality of the diagnostic and treatment programme in Cyprus.

Therefore, Cyprus has virtually reached the TB elimination phase in its native-born population (figure 3), even though a limited number of cases in foreign-born individuals brought the overall figure above the TB elimination threshold.

Voniatis et al. [96] identified the following interventions as necessary to consolidate the results achieved. 1) Focus on the management of vulnerable populations (especially migrants) with constant monitoring of the capacity to ensure early detection and effective treatment. 2) Design of a specifically funded TB elimination plan with objectives ad targets. 3) Implementation of the electronic LTBI register with evaluation of completion rates. 4) Approval of the planned universal coverage and social protection schemes to protect the most vulnerable people.

The Cypriot study, although small and not representative of the European situation, was important as proof of concept that TB elimination is feasible [96].

Oman

Oman represents the second country to be formally evaluated under the TB elimination perspective [97]. Located among the Arabian Gulf Countries, the country (and the whole region) have a TB epidemiology within the pre-elimination phase, with low incidence of TB among natives and the majority of cases occurring among foreign-born people (representing about half of the total population: 1986226 out of 4 414051 in 2016).

Effective TB control programmes are in place, including the eight core elimination activities described above.

Government commitment is testified by the important budget (US\$ 1.6 billion) allocated for health, representing $13.6 \%$ of the state budget. Oman has developed a draft plan for TB elimination.

The study described the trajectory of Oman towards TB elimination between 2000 and 2016. Overall, in 2016 Oman notified 344 cases of TB, 190 among natives and 154 among foreign-born cases.

The TB notification rate (all cases, as well as sputum smear positive cases) declined among Oman-born individuals from 2010 to 2016, while the decline among foreign-born sputum smear positive patients started in 2014 (figure 4). The average decline of TB cases in Oman was $-2 \%$ per year.

In 2016 the incidence of notified sputum smear positive cases was 38.1 per million population and that of all forms of TB was 77.9 per million. For Oman-born cases, the incidence was 28.4 and 78.2 per million, respectively, and among foreign-born cases was 49.8 and 77.5, respectively.

Oman reported excellent treatment outcomes (88\% success in the 2015 cohort, $7 \%$ death rate and only $3 \%$ lost to follow-up) and a low treatment delay (half of the cases diagnosed within 1 month from the onset of symptoms). 
FIGURE 4 Decline of the case notification rate of tuberculosis (TB) towards the elimination threshold (one case per million population) in Oman. $\mathrm{SS}^{+}$: sputum smear positive. Reproduced from [97] with permission.

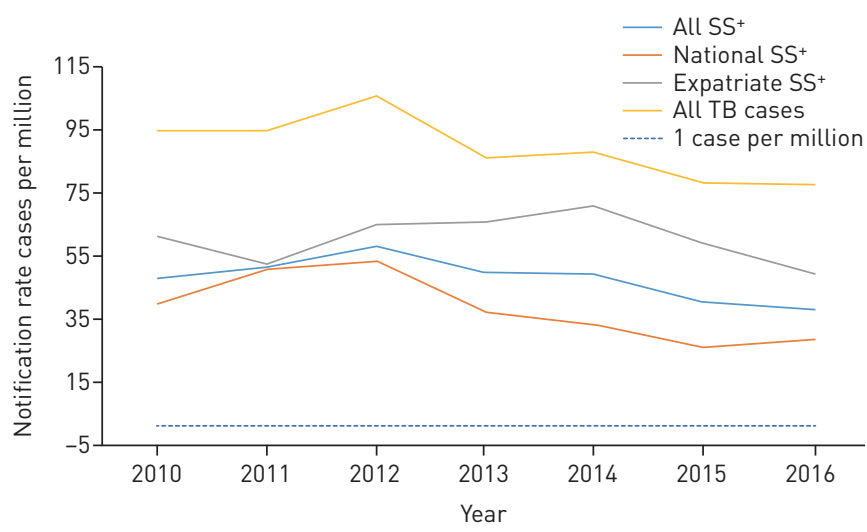

Mortality, as in the majority of low TB incidence countries, was only apparently high as it affected the elderly with co-morbidities (and TB was not the primary cause of death).

As in other Gulf Countries the TB/HIV co-infection rate is very low (12 (3.5\%) cases in 2016, all under antiretroviral treatment) and MDR-TB was limited to six cases in 2016 (an average of 4.4 cases per year during the study period).

Health authorities in Oman are trying to increase contact tracing: contacts are systematically screened using interferon gamma release assays or the tuberculin skin test. In 2016, 70\% of the 1473 registered contacts among Omani-born persons were screened, $142(13.8 \%)$ had a positive LTBI test and none had active TB. Among the 455 registered foreign-born contacts, 410 (90\%) were screened and 80 (19.5\%) had LTBI.

Looking at the progress towards TB elimination (figure 4), in 2011 the incidence among foreign-born patients started exceeding that of natives; incidence rates for both foreign-born cases and nationals have presently reached a "plateau".

The Oman health authorities have committed to further strengthen the existing TB elimination plan and its LTBI component. In particular, more efforts will be performed to define priority groups for LTBI diagnosis and treatment (e.g. foreign born beyond contacts) [97].

\section{A regional approach: the experience of Latin America}

Several countries in Latin America and the Caribbean have low TB incidence or are approaching this threshold [1, 71, 72]: Costa Rica, Cuba, Jamaica, Puerto Rico and English speaking islands have an incidence of $<10$ cases per 100000 population while Chile and Trinidad and Tobago have between 10 and 20 cases per 100000 inhabitants.

Following a meeting organised in July 2016 involving representatives of the Asociación Latinoamericana de Tórax (ALAT), the European Respiratory Society (ERS) and the Panamerican Health Organization/ WHO in Santiago, Chile, a plan was designed to pursue TB elimination in the region considering: 1) the epidemiological and programmatic heterogeneity among countries; 2) the lack of published scientific local data; and 3) the need for a local adaptation of the TB elimination framework.

The Hoja de Ruta para la Eliminación de la Tuberculosis en Latinoamérica y el Caribe 2016-2025 [71, 72] identified eight components and specific actions to achieve TB elimination in the region, as summarised in tables 4 and 5. Tables 4 and 5 show the indicators identified by the Latin American countries to measure

TABLE 4 Main indicators recommended for Latin American and Caribbean countries

Impact indicators

1. Reduction in number of TB deaths compared with 2015

2. Reduction in TB incidence rate compared with 2015

3. TB-affected families facing catastrophic costs due to TB
Milestones

$2020 \quad 2025$
Targets

SDG 2030 End TB 2035

Data are presented as \%. SDG: sustainable development goals; TB: tuberculosis. Reproduced from [71] with permission. 
TABLE 5 Top 10 implementation indicators of the End TB Strategy

Implementation indicators

Recommended

target level \%

\section{TB treatment coverage}

2. TB treatment success rate

3. Percentage of TB-affected households that experience catastrophic costs due to TB

4. Percentage of newly notified TB patients diagnosed using WHO recommended rapid tests

5. Latent TB infection treatment coverage

6. Contact investigation coverage

7. Drug susceptibility coverage for TB patients

8. Treatment coverage, new TB drugs

9. Documentation of HIV status among TB patients

TB: tuberculosis; WHO: World Health Organization. Reproduced from [71] with permission.

the trajectory towards TB elimination. At present it is too early to know if countries will be able to meet them. Although a systematic review evaluating to what extent the eight core areas for TB elimination are covered is beyond the scope of the present review, it is clear that several areas need to be further strengthened. In particular a strong political commitment is necessary at a country level (ensuring adequate financial and human resources, particularly from the public health and community perspective), with improved surveillance and active case finding in risk groups. Furthermore, increased international collaboration and enhancement of the existing programmes targeting big cities and their specific problems will be beneficial. Still, the population access to rapid testing, shorter treatments for LTBI (e.g. rifapentine is not yet registered in Europe) and availability of new drugs (bedaquiline and delamanid) is largely sub-optimal in several low TB incidence countries.

Furthermore, a TB research network was created (the ALAT/ERS LATSINTB project) to tackle the evidence gap, based on the positive results achieved by previous informal collaboration between ERS and Latin American researchers [28, 32, 33, 47, 49, 63, 71, 72, 84, 85, 98-100]. These, as well as similar initiatives, are necessary and properly coordinated with well-designed national research plans.

\section{The TB elimination plan in Brazil}

According to $\mathrm{WHO}$, Brazil is one of the countries included in the set of priority countries for TB and TB-HIV. In 2016, 67000 people fell ill with TB and of these, 4500 died. Besides these alarming numbers, epidemiological trends show a decrease in incidence rates, from 51.8 cases per 100000 inhabitants in 1990 to 33.5 cases per 100000 inhabitants in 2017 , while mortality rates decreased from 3.6 to 2.1 cases per 100000 population in the same period. In spite of these positive trends and because of the continental size of the country and different socio-economic characteristics in different regions, TB epidemiological indicators have important intra-country differences.

Following the WHO End TB Strategy, the National TB Control Programme in Brazil proposed a National Plan to End TB as a Public Health Problem in 2017. The main aim of this plan is to reduce incidence rates to levels $<10$ cases per 100000 population and mortality rates to $<1$ case per 100000 population by 2035, using the same pillars as the End TB Strategy.

The main strengths of the TB Brazilian programme are the universal diagnostic coverage, the treatment managed via the public health system, the decentralised plan for TB patients follow-up, the active involvement of both the civil society and the REDE-TB (the national research network) and the capacity to achieve some of the WHO targets (e.g. TB detection rates at the level of $87 \%$ ). The most important future challenges will be more focused on improving TB treatment outcomes, implementing new diagnosis and treatment technologies and an innovative approach to the disease from a social perspective [101, 102].

\section{Towards TB elimination in Mexico}

In 2017 Mexico reported 21716 incident TB cases in 2017 with only 213 drug-resistant cases. Between 1997 and 2017 the annual TB incidence decline was around 30\%, corresponding to an average annual decline of $1.7 \%$, which is more pronounced during the first 10 years (1997-2007) of the last 20 years (1997-2017). 
Interestingly, out of a total of 32 states in Mexico, in 2017 one-third reported a TB incidence rate of $<10$ cases per 100000 population, one-third between 10 and 20 cases per 100000 population and the remaining third $>20$ cases per 100000 population.

Formally, one- to two-thirds of the Mexican states have low TB incidence; the previous WHO threshold value defining low TB incidence countries was 20 cases per 100000 population.

This implies that the Mexican National TB Control Programme needs to plan a double velocity to approach the TB pre-elimination phase.

Although a TB elimination plan has not been developed yet, national authorities are working to update the national guidelines in order to focus them more on LTBI management.

The main strengths of the Mexican programme are the universal access to TB diagnosis and treatment services (including MDR-TB), the availability of first- and second-line drugs of proven quality, as well as the availability of national guidelines for the diagnosis and treatment of TB, and a quality surveillance system.

The core challenges the Mexican health authorities need to face in the future will be mainly focused on the improvement of: 1) TB active case finding (particularly of drug-resistant cases); 2) access to rapid diagnostics; and 3) management of comorbidities (including diabetes). In addition further actions will be necessary, such as to: 1) update the LTBI management guidelines (e.g. access to new diagnostics and new shorter regimens; 2) re-define the risk groups to test them in low incidence states; 3) expand the network of laboratories performing drug susceptibility testing for second-line drugs; and 4) to enhance the patient-centred approach to reduce diagnosis delay and improve treatment outcomes.

\section{Conclusions}

In summary, although epidemiological plausibility for TB elimination exists, a comprehensive effort is needed by national TB programmes to implement the eight core activities in different settings, including Europe, the Gulf Countries and Latin America, as well as other regions. At the same time new prevention, diagnostic and treatment tools are also necessary in order to increase the speed of the present TB incidence decline.

Acknowledgements: This article is part of the European Respiratory Society/Latino-American Society of Respiratory Medicine and the European Respiratory Society/Brazilian Society of Pulmonology collaborative tuberculosis projects. The authors wish to thank Tania Herrera (Public Health Specialist, National TB Programme Director, Ministry of Health, Chile), Ana Paula Santos (State University of Rio de Janeiro and Federal University of Rio de Janeiro, Brazil) and Marcela Muñoz Torrico (INER Institute, Mexico City, Mexico) for the useful comments to the revised version of the manuscript.

Conflict of interest: None declared.

\section{References}

World Health Organization. Global tuberculosis report 2017. Geneva, World Health Organization, 2017.

Migliori GB, Sotgiu G, Gandhi NR, et al. Drug resistance beyond extensively drug-resistant tuberculosis: individual patient data meta-analysis. Eur Respir J 2013; 42: 169-179.

3 Falzon D, Gandhi N, Migliori GB, et al. Resistance to fluoroquinolones and second-line injectable drugs: impact on multidrug-resistant TB outcomes. Eur Respir J 2013; 42: 156-168.

Uplekar M, Weil D, Lonnroth K, et al. WHO’s new end TB strategy. Lancet 2015; 385: 1799-1801.

Sotgiu G, Spanevello A, Migliori GB. History of tuberculosis and drug resistance. N Engl J Med 2013; 368: 88-89.

Centis R, D'Ambrosio L, Zumla A, et al. Shifting from tuberculosis control to elimination: Where are we? What are the variables and limitations? Is it achievable? Int J Infect Dis 2017; 56: 30-33.

7 Migliori GB, Sotgiu G, Lange C, et al. Extensively drug-resistant tuberculosis: back to the future. Eur Respir J 2010; 36: 475-477.

8 Styblo K, Meijer J, Sutherland I. Tuberculosis Surveillance Research Unit Report No. 1: the transmission of tubercle bacilli; its trend in a human population. Bull Int Union Tuberc 1969; 42: 5-104.

9 Veen J, Migliori GB, Raviglione M, et al. Harmonisation of TB control in the WHO European region: the history of the Wolfheze Workshops. Eur Respir J 2011; 37: 950-959.

10 World Health Organization. Treatment of tuberculosis: guidelines. 4th Edn. Geneva, World Health Organization, 2010.

11 TB CARE I. International Standards for Tuberculosis Care. 3rd Edn. The Hague, TB CARE I, 2014.

12 Migliori GB, Zellweger JP, Abubakar I, et al. European Union standards for tuberculosis care. Eur Respir J 2012; 39: 807-819.

13 Migliori GB, Sotgiu G, Rosales-Klintz S, et al. ERS/ECDC Statement: European Union Standards for Tuberculosis Care, 2017 update. Eur Respir J 2018; 51: 1702678.

14 Sotgiu G, Beer N, Aliberti S, et al. Fighting tuberculosis in the EU/EEA: towards the new European Union standards on tuberculosis care. Eur Respir J 2016; 48: 1278-1281.

15 van der Werf MJ, Sandgren A, D'Ambrosio L, et al. The European Union standards for tuberculosis care: do they need an update? Eur Respir J 2014; 43: 933-942. 

and control after 2015. www.who.int/tb/strategy/End_TB_Strategy.pdf?ua=1 Date last updated: March 2016; date last accessed: May 8, 2018.

17 Sotgiu G, Mauch V, Migliori GB, et al. Evidence-based, agreed-upon health priorities to remedy the tuberculosis patient's economic disaster. Eur Respir J 2014; 43: 1563-1566.

18 Lönnroth K, Migliori GB, Abubakar I, et al. Towards tuberculosis elimination: an action framework for low-incidence countries. Eur Respir J 2015; 45: 928-952.

19 World Health Organization. Framework for tuberculosis elimination in low-incidence countries. Geneva, World Health Organization, 2016.

20 Gilpin C, Korobitsyn A, Migliori GB, et al. The World Health Organization Standards for tuberculosis care and management. Eur Respir J 2018; 51: 1800098.

21 Getahun H, Matteelli A, Abubakar I, et al. Management of latent Mycobacterium tuberculosis infection: WHO guidelines for low tuberculosis burden countries. Eur Respir J 2015; 46: 1563-1576.

22 World Health Organization. Latent tuberculosis infection: updated and consolidated guidelines for programmatic management. Geneva, World Health Organization, 2018.

23 Creswell J, Raviglione M, Ottmani S, et al. Tuberculosis and noncommunicable diseases: neglected links and missed opportunities. Eur Respir J 2011; 37: 1269-1282.

24 Migliori GB, Lienhardt C, Weyer K, et al. Ensuring rational introduction and responsible use of new TB tools: outcome of an ERS multisector consultation. Eur Respir J 2014; 44: 1412-1417.

25 Rudgard WE, das Chagas NS, Gayoso R, et al. Uptake of governmental social protection, and financial hardship during drug-resistant tuberculosis treatment in Rio de Janeiro, Brazil. Eur Respir J 2018; 51: 1800274.

26 Petersen E, Khamis F, Migliori GB, et al. De-isolation of patients with pulmonary tuberculosis after start of treatment - clear, unequivocal guidelines are missing. Int J Infect Dis 2017; 56: 34-38.

27 Broekmans JF, Migliori GB, Rieder HL, et al. European framework for tuberculosis control and elimination in countries with a low incidence. Recommendations of the World Health Organization (WHO), International Union Against Tuberculosis and Lung Disease (IUATLD) and Royal Netherlands Tuberculosis Association (KNCV) Working Group. Eur Respir J 2002; 19: 765-775.

28 Duarte R, Silva DR, Rendon A, et al. Eliminating tuberculosis in Latin America: making it the point. J Bras Pneumol. 2018; 44: 73-76.

29 Dye C, Glaziou P, Floyd K, et al. Prospects for tuberculosis elimination. Annu Rev Public Health 2013; 34 271-286.

30 Carter DJ, Glaziou P, Lönnroth K, et al. The impact of social protection and poverty elimination on global tuberculosis incidence: a statistical modelling analysis of Sustainable Development Goal 1. Lancet Glob Health 2018; 6: e514-e522.

31 Migliori GB, Garcia-Basteiro AL. Predicting the effect of improved socioeconomic health determinants on the tuberculosis epidemic. Lancet Glob Health 2018; 6: e475-e476.

32 Silva DR, Sotgiu G, D’Ambrosio L, et al. Diagnostic performances of the Xpert MTB/RIF in Brazil. Respir Med 2018; 134: 12-15.

33 García-Basteiro AL, DiNardo A, Saavedra B, et al. Point of care diagnostics for tuberculosis. Pulmonology 2018; 24: 73-85.

34 World Health Organization. Compendium of WHO guidelines and associated standards: ensuring optimum delivery of the cascade of care for patients with tuberculosis. http://apps.who.int/iris/bitstream/10665/259180/1/ 9789241512572-eng.pdf Date last updated: 2017; date last accessed: March 29, 2018.

35 Weyer K, Mirzayev F, Migliori GB, et al. Rapid molecular TB diagnosis: evidence, policy making and global implementation of Xpert MTB/RIF. Eur Respir J 2013; 42: 252-271.

36 Wallis RS, Maeurer M, Mwaba P, et al. Tuberculosis - advances in development of new drugs, treatment regimens, host-directed therapies, and biomarkers. Lancet Infect Dis 2016; 16: e34-e46.

37 Schito M, Migliori GB, Fletcher HA, et al. Perspectives on advances in tuberculosis diagnostics, drugs, and vaccines. Clin Infect Dis 2015; 61: Suppl. 3, S102-S118.

38 World Health Organization. WHO treatment guidelines for drug-resistant tuberculosis 2016 update. Geneva World Health Organization, 2016.

39 Falzon D, Schünemann HJ, Harausz E, et al. World Health Organization treatment guidelines for drug-resistant tuberculosis, 2016 update. Eur Respir J 2017; 49: 1602308.

40 Ahmad Khan F, Salim MAH, du Cros P, et al. Effectiveness and safety of standardised shorter regimens for multidrug-resistant tuberculosis: individual patient data and aggregate data meta-analyses. Eur Respir J 2017; 50: 1700061.

41 Sotgiu G, Tiberi S, D'Ambrosio L, et al. Faster for less, the new 'shorter' regimen for multidrug-resistant tuberculosis. Eur Respir J 2016; 48: 1503-1507.

42 van der Werf MJ, Hollo V, Ködmön C, et al. Eligibility for shorter treatment of multidrug-resistant tuberculosis in the European Union. Eur Respir J 2017; 49: 1601992.

43 Heldal E, Van Deun A, Chiang C-Y, et al. Shorter regimens for multidrug-resistant tuberculosis should also be applicable in Europe. Eur Respir J 2017; 49: 1700228.

44 van der Werf MJ, Ködmön C, Catchpole M. Shorter regimens for multidrug-resistant tuberculosis should also be applicable in Europe. Eur Respir J 2017; 49: 1700463.

45 Javaid A, Ahmad N, Hayat Khan A, et al. Applicability of World Health Organization's recommended new shorter regimen in a multidrug-resistant tuberculosis high burden country. Eur Respir J 2017; 49: 1601967.

46 Van Deun A, Chiang C-Y. Shortened MDR-TB regimens overcome low-level fluoroquinolone resistance. Eur Respir J 2017; 49: 1700223.

47 Dalcolmo M, Gayoso R, Sotgiu G, et al. Resistance profile to the drugs composing the 'shorter' regimen for multidrug-resistant TB in Brazil, 2000-2015. Eur Respir J 2017; 49: 1602309.

48 Chee CBE, KhinMar KW, Sng LH, et al. The shorter multidrug-resistant tuberculosis treatment regimen in Singapore: are patients from South-East Asia eligible? Eur Respir J 2017; 50: 1700753

49 Munoz-Torrico M, Salazar MA, Millán MJM, et al. Eligibility for the shorter regimen for multidrug-resistant tuberculosis in Mexico. Eur Respir J 2018; 51: 1702267. 
Migliori GB, Eker B, Richardson MD, et al. A retrospective TBNET assessment of linezolid safety, tolerability and efficacy in MDR-TB. Eur Respir J 2009; 34: 387-393.

Sotgiu G, Lange C, Richardson MD, et al. Comment on: daily $300 \mathrm{mg}$ dose of linezolid for the treatment of intractable multidrug-resistant and extensively drug-resistant tuberculosis. J Antimicrob Chemother 2009; 64: 879-883.

Villar M, Sotgiu G, D'Ambrosio L, et al. Linezolid safety, tolerability and efficacy to treat multidrug- and extensively drug-resistant tuberculosis. Eur Respir J 2011; 38: 730-733. 770-772. treating MDR-TB and XDR-TB: systematic review and meta-analysis. Eur Respir J 2012; 40: 1430-1442.

Sotgiu G, Centis R, D’Ambrosio L, et al. Linezolid to treat extensively drug-resistant TB: retrospective data are confirmed by experimental evidence. Eur Respir J 2013; 42: 288-290.

Sotgiu G, Pontali E, Migliori GB. Linezolid to treat MDR-/XDR-tuberculosis: available evidence and future scenarios. Eur Respir J 2015; 45: 25-29.

Sotgiu G, Centis R, D'Ambrosio L, et al. Low minimal inhibitory concentrations of linezolid against multidrug-resistant tuberculosis strains. Eur Respir J 2015; 45: 287-289.

Bolhuis MS, Tiberi S, Sotgiu G, et al. Linezolid tolerability in multidrug-resistant tuberculosis: a retrospective study. Eur Respir J 2015; 46: 1205-1207.

Bolhuis MS, Tiberi S, Sotgiu G, et al. Is there still room for therapeutic drug monitoring of linezolid in patients with tuberculosis? Eur Respir J 2016; 47: 1288-1290.

Tiberi S, Sotgiu G, D'Ambrosio L, et al. Comparison of effectiveness and safety of imipenem/clavulanate- versus meropenem/clavulanate-containing regimens in the treatment of MDR- and XDR-TB. Eur Respir J 2016; 47: $1758-1766$.

Tiberi S, Payen MC, Sotgiu G, et al. Effectiveness and safety of meropenem/clavulanate-containing regimens in the treatment of MDR- and XDR-TB. Eur Respir J 2016; 47: 1235-1243.

Tiberi S, D'Ambrosio L, De Lorenzo S, et al. Ertapenem in the treatment of multidrug-resistant tuberculosis: first clinical experience. Eur Respir J 2016; 47: 333-336.

Dalcolmo M, Gayoso R, Sotgiu G, et al. Effectiveness and safety of clofazimine in multidrug-resistant tuberculosis: a nationwide report from Brazil. Eur Respir J. 2017; 49: 1602445.

Borisov SE, Dheda K, Enwerem M, et al. Effectiveness and safety of bedaquiline-containing regimens in the treatment of MDR- and XDR-TB: a multicentre study. Eur Respir J. 2017; 49: 1700387.

Tadolini M, Garcia-Prats AJ, D'Ambrosio L, et al. Compassionate use of new drugs in children and adolescents with multidrug-resistant and extensively drug-resistant tuberculosis: early experiences and challenges. Eur Respir J 2016; 48: 938-943.

Maryandyshev A, Pontali E, Tiberi S, et al. Bedaquiline and delamanid combination treatment of 5 patients with pulmonary extensively drug-resistant tuberculosis. Emerg Infect Dis 2017; 23: 1718-1721.

Tadolini M, Lingtsang RD, Tiberi S, et al. First case of extensively drug-resistant tuberculosis treated with both delamanid and bedaquiline. Eur Respir J 2016; 48: 935-938.

Pontali E, Sotgiu G, D'Ambrosio L, et al. Bedaquiline and multidrug-resistant tuberculosis: a systematic and critical analysis of the evidence. Eur Respir J 2016; 47: 394-402.

Pontali E, D'Ambrosio L, Centis R, et al. Multidrug-resistant tuberculosis and beyond: an updated analysis of the current evidence on bedaquiline. Eur Respir J 2017; 49: 1700146.

Pontali E, Sotgiu G, Tiberi S, et al. Cardiac safety of bedaquiline: a systematic and critical analysis of the evidence. Eur Respir J 2017; 50: 1701462.

Rendon A, Fuentes Z, Torres-Duque CA, et al. Roadmap for tuberculosis elimination in Latin American and Caribbean countries: a strategic alliance. Eur Respir J 2016; 48: 1282-1287.

Torres-Duque CA, Fuentes Alcalá ZM, Rendón A, et al. Roadmap for tuberculosis elimination in Latin America and the Caribbean. Arch Bronconeumol 2018; 54: 7-9.

D'Ambrosio L, Dara M, Tadolini M, et al. Tuberculosis elimination: theory and practice in Europe. Eur Respir J 2014; 43: 1410-1420.

Ministry of Health of the Russian Federation, World Health Organization. Moscow declaration to End TB. First WHO global ministerial conference Ending TB in the sustainable development era: a multisectoral response. www.who.int/tb/features_archive/Moscow_Declaration_to_End_TB_final_ENGLISH.pdf?ua=1 Date last updated: November 17, 2017. Date last accessed: March 22, 2018.

Blasi F, Matteelli A, Sotgiu G, et al. Moving towards tuberculosis elimination: a call for action from Italy and a possible model for other low tuberculosis incidence countries. Eur Respir J 2017; 49: 1602242.

Dara M, Solovic I, Sotgiu G, et al. Call for urgent actions to ensure access to early diagnosis and care of tuberculosis among refugees: statement of the European Respiratory Society and the European Region of the International Union Against Tuberculosis and Lung Disease. Eur Respir J 2016; 47: 1345-1347.

Dara M, Solovic I, Sotgiu G, et al. Tuberculosis care among refugees arriving in Europe: a ERS/WHO Europe Region survey of current practices. Eur Respir J 2016; 48: 808-817.

Dara M, Sulis G, Centis R, et al. Cross-border collaboration for improved tuberculosis prevention and care: policies, tools and experiences. Int J Tuberc Lung Dis 2017; 21: 727-736.

Dara M, de Colombani P, Petrova-Benedict R, et al. Minimum package for cross-border TB control and care in the WHO European region: a Wolfheze consensus statement. Eur Respir J 2012; 40: 1081-1090.

Noeske J, Foe JL, Kuaban C. Cameroon's MDR-TB treatment programme jeopardised by cross-border migration. Eur Respir J 2016; 47: 684-686.

Matteelli A, Lönnroth K, Mosca D, et al. Cameroon's MDR-TB treatment programme jeopardised by cross-border migration. Eur Respir J 2016; 47: 686-688.

Dara M, Gushulak BD, Posey DL, et al. The history and evolution of immigration medical screening for tuberculosis. Expert Rev Anti Infect Ther 2013; 11: 137-146.

Holguin F, Moughrabieh MA, Ojeda V, et al. Respiratory health in migrant populations: a crisis overlooked. Ann Am Thorac Soc 2017; 14: 153-159. 
UNHCR - The UN Refugee Agency. Refugees/migrants emergency response - Mediterranean. http://data2.unhcr. org/en/situations/mediterranean Date last updated: May 2018; date last accessed: May 8, 2018.

Nicolai T, Fuchs O, von Mutius E. Caring for the wave of refugees in Munich. $N$ Engl J Med 2015; 373: 1593-1595.

Muñoz-Torrico M, Caminero Luna J, Migliori GB, et al. Comparison of bacteriological conversion and treatment outcomes among MDR-TB patients with and without diabetes in Mexico: Preliminary data. Rev Port Pneumol (2006) 2017; 23: 27-30.

Muñoz-Torrico M, Caminero-Luna J, Migliori GB, et al. Diabetes is associated with severe adverse events in multidrug-resistant tuberculosis. Arch Bronconeumol 2017; 53: 245-250.

World Health Organization. Consensus meeting report: development of a Target Product Profile (TPP) and a framework for evaluation for a test for predicting progression from tuberculosis infection to active disease. Geneva, World Health Organization, 2017.

Swindells S, Ramchandani R, Gupta A, et al. One month of rifapentine/isoniazid to prevent TB in people with HIV: Brief-TB/A5279. Abstract 37LB. www.croiconference.org/sessions/one-month-rifapentineisoniazid-preventtb-people-hiv-brief-tba5279 Date last updated: March 2018; date last accessed: May 8, 2018.

Diel R, Vandeputte J, de Vries G, et al. Costs of tuberculosis disease in the European Union: a systematic analysis and cost calculation. Eur Respir J 2014; 43: 554-565.

Diel R, Rutz S, Castell S, et al. Tuberculosis: cost of illness in Germany. Eur Respir J 2012; 40: 143-151. and households. Geneva, World Health Organization, 2009

Yuen CM, Amanullah F, Dharmadhikari A, et al. Turning off the tap: stopping tuberculosis transmission through active case-finding and prompt effective treatment. Lancet 2015; 386: 2334-2343.

Rieder HL, Watson JM, Raviglione MC, et al. Surveillance of tuberculosis in Europe. Working Group of the World Health Organization (WHO) and the European Region of the International Union Against Tuberculosis and Lung Disease (IUATLD) for uniform reporting on tuberculosis cases. Eur Respir J 1996; 9: 1097-1104.

Uplekar M, Atre S, Wells WA, et al. Mandatory tuberculosis case notification in high tuberculosis-incidence countries: policy and practice. Eur Respir J 2016; 48: 1571-1581.

Voniatis C, Migliori GB, Voniatis M, et al. Tuberculosis elimination: dream or reality? The case of Cyprus. Eur Respir J 2014; 44: 543-546.

Al Yaquobi F, Al-Abri S, Al-Abri B, et al. Tuberculosis elimination: a dream or a reality? The case of Oman. Eur Respir J 2018; 51: 1702027.

D’Ambrosio L, Bothamley G, Caminero Luna JA, et al. Team approach to manage difficult-to-treat TB cases: experiences in Europe and beyond. Pulmonology 2018; 24: 132-141.

Tiberi S, Muñoz-Torrico M, Duarte R, et al. New drugs and perspectives for new anti-tuberculosis regimens. Pulmonology 2018; 24: 86-98.

0 Duarte R, Lönnroth K, Carvalho C, et al. Tuberculosis, social determinants and co-morbidities (including HIV). Pulmonology 2018; 24: 115-119.

101 Coordenação-Geral do Programa Nacional de Controle da Tuberculose. Departamento de Vigilância das Doenças Transmissíveis. Secretaria de Vigilância em Saúde. Ministério da Saúde. Programa Nacional de Controle da Tuberculose. http://portalarquivos2.saude.gov.br/images/pdf/2018/marco/19/APRES-PADRAO-JAN-2018-REDU ZIDA.pdf Date last updated: March 2018; date last accessed: May 8, 2018.

102 Ministério da Saúde. Secretaria de Vigilância em Saúde. Departamento de Vigilância das Doenças Transmissíveis Brasil Livre da Tuberculose: Plano Nacional pelo Fim da Tuberculose como Problema de Saúde Pública. Brasília, Ministério da Saúde, 2017. 